Teil 3

Archivierung und Regulierung 



\title{
Licht und Schatten in der akademischen Medienindustrie
}

\author{
Georg Fischer"
}

Die Transformation hin zu einer digital vernetzten Welt betrifft nicht nur die klassische Unterhaltungsindustrie, sondern auch einen Industriezweig, den ich als akademische Medienindustrie bezeichne. Darunter verstehe ich einen wirtschaftlichen Sektor, der als Geschäftsmodell in erster Linie die Distribution, Vermarktung und Verwertung wissenschaftlicher Publikationen betreibt - Publikationen wohlgemerkt, die fast ausschließlich von Wissenschaftler:innen selbst konzipiert und angefertigt werden, nicht von den Verlagen.

Involviert in das Geschäft sind vor allem Großverlage wie Springer Nature, Elsevier oder Wiley. Sie bieten wissenschaftliche Publikationen ganz klassisch in papierner Form als Buch oder Zeitschrift sowie zunehmend in de-materialisierten, digital kopierbaren Formaten an. Nachgefragt werden diese Texte von Universitäten und Forschungseinrichtungen, Bibliotheken und Gedächtnisinstitutionen sowie natürlich von Gruppen, die in Forschung und Lehre beteiligt sind, etwa Studierende, Dozent:innen und Forscher:innen; daneben von Journalist:innen, freischaffenden Intellektuellen oder anderweitig interessierten Personen sowie auch wissenschaftsexternen Akteur:innen aus Politik, Wirtschaft oder Industrie.

Wissenschaftler:innen verfassen die Texte nicht nur, sie sorgen zumeist im Sinne des peer review auch im Wesentlichen für ihre inhaltliche und oft auch formale Qualitätskontrolle, ohne dafür von Verlagen bezahlt zu werden. Sie tun dies im Rahmen ihrer Tätigkeit als Wissenschaftler:innen, die dafür verwendeten Ressourcen stammen also in aller Regel aus öffentlichen Mitteln. Zwar sind Wissenschaftler:innen nicht als homogene Gruppe zu verstehen, die alle nach den gleichen Zielen streben oder sogar mit den gleichen Ressourcen ausgestattet sind. Sie sind aber alle darauf angewiesen, dass ihre Forschungsergebnisse von Kolleg:innen wahrgenommen und weiterentwickelt werden, nicht nur innerhalb enger disziplinärer

* Dr. Georg Fischer ist Soziologe und arbeitet als Redakteur bei iRights.info. Seine Forschungsinteressen gelten empirischer Urheberrechtsforschung, vor allem Verwertungsgesellschaften, fremdreferenzieller Musik und Fragen des geistigen Eigentums in der Wissenschaft. 
Kreise, sondern auch in internationalen Zusammenhängen und wissenschaftsexternen Kontexten. Um sich auf ihre Forschungs- und Lehrtätigkeit konzentrieren zu können, verlassen sie sich daher auf die Verlage, die die Publikation und Distribution ihrer Texte übernehmen.

Das Urheberrecht fungiert nicht nur in der Unterhaltungs-, sondern auch in der akademischen Medienindustrie als bürokratisch-technische Grundlage der Wertschöpfung, auf dessen Basis Mediengüter veröffentlicht, verwertet und lizenziert werden. Die Wege und Techniken der Zugänglichmachung wissenschaftlicher Literatur sind das Hauptthema dieses Aufsatzes. Ich möchte dafür allerdings nicht primär die urheberrechtlichen Maßgaben erläutern, in die die wissenschaftlichen Großverlage der akademischen Medienindustrie eingebunden sind, sondern nach den sich ergebenden Konsequenzen für den Bereich des wissenschaftlichen Publizierens fragen. Ich fokussiere dafür nicht die offiziell sichtbaren, hell ausgeleuchteten Publikationsformen der wissenschaftlichen Großverlage. Meine Untersuchung beginnt dort, wo das Juristische in eine Grauzone gelangt und die tägliche Praxis wissenschaftlicher Publikationen im Schatten liegt. Dieser Schatten ist metaphorisch zu verstehen: Gemeint ist ein Bereich, der von den Akteur:innen selbst so verdunkelt oder anderweitig gestaltet ist, dass man ihn nicht ohne weiteres einsehen oder betreten kann.

Das Internet bietet eine Vielzahl an solchen Orten, die im Schatten liegen, wie zum Beispiel das Darknet, Filesharing-Plattformen für Musik, Videos und Belletristik - und eben auch Websites für wissenschaftliche Publikationen. Diese sogenannten Schattenbibliotheken existieren in technischer Hinsicht, weil es in den vergangenen Jahrzehnten einen enormen Schub in der technischen Reproduzierbarkeit von medialen Konsumgütern gegeben hat. In dessen Folge konnten sich die Inhalte (wissenschaftliche Texte) von ihren medialen Trägern (Papier) lösen und in neuen digitalen, leichter zu kopierenden Formaten (PDF, ePUB) angeboten werden. Die digitale Kopierbarkeit bietet die technische Grundlage für die Zusammenstellung großer, umfassender Textkorpora. Doch Schattenbibliotheken sind nicht nur ein technisches Phänomen, sie bedienen freilich auch eine gesellschaftliche Nachfrage: nämlich nach einem einfachen, kostenlosen und möglichst barrierefreien Zugang zu wissenschaftlichen Erkenntnissen. Dieses Bedürfnis wiederum konfligiert mit den urheberrechtlichen Maßgaben wissenschaftlicher Texte und den daran gekoppelten wirtschaftlichen Verwertungsmechanismen auf Seiten der Großverlage.

Ziel dieses Artikels ist es daher, Schattenbibliotheken als urheberrechtlich konfliktäre Praxis zwischen Akteur:innen mit verschiedenen Interessen darzustellen und die diesbezügliche Dynamik historisch einzuordnen. Für diesen Zweck nutze ich das Wechselspiel zwischen Steigerung und 
Suspension technischer Reproduzierbarkeit als theoretisches Konstrukt, um die Konfliktlinie um das Urheberrecht beim wissenschaftlichen Publizieren genauer herauszuarbeiten und im Sinne von historischen Kipppunkten zu interpretieren. Darauf aufbauend veranschauliche ich anhand von \#canIhazPDF, Ubuweb, AAARG, Library Genesis und Science Hub die Genese und Vielfalt wissenschaftlicher Schattenbibliotheken. Abschließend diskutiere ich die Frage, inwiefern der Aufstieg des Prinzips der Schattenbibliothek als Tipping Point, also als Umschlags- oder Kipppunkt in der Entwicklung der akademischen Medienindustrie verstanden werden kann.

\section{Der Kampf um die Hoheit zur technischen Reproduzierbarkeit}

Die medienindustrielle Dynamik, in der Schattenbibliotheken agieren, möchte ich genauer umreißen. Dazu schlage ich vor, sie als Kampf um die Hoheit zur technischen Reproduzierbarkeit zu begreifen und im Sinne des Kipppunkt-Konzepts zu spezifizieren. Stichwortgeber dafür ist Walter Benjamin, der mit seinem bereits in den 1930er Jahren erschienenen Aufsatz „Das Kunstwerk im Zeitalter seiner technischen Reproduzierbarkeit“ das Phänomen massenhaft herstellbarer Kopien und dadurch resultierende Implikationen eindrucksvoll beschrieb. ${ }^{1}$ Zwar hatte Benjamin nicht gerade urheberrechtliche Probleme vor Augen, als er seinen einflussreichen Aufsatz verfasste, doch er war durchaus fasziniert von den Möglichkeiten der Vervielfältigung von physischen Objekten und medialen Datenträgern wie Fotografien und Filmaufnahmen.

Um in dieser Faszination für die Steigerung technischer Reproduzierbarkeit nicht den Blick für gegenläufige Entwicklungen zu verlieren, forderte der Medienwissenschaftler Jens Schröter viele Jahre nach Benjamin, stärker die Mechanismen der „Nicht-Reproduzierbarkeit“ zu berücksichtigen. In diesem Sinne plädiert Schröter dafür, die Konfigurationen technischer Reproduzierbarkeit, also die „unterschiedlichen Stufungen und Verteilungen von Reproduzierbarkeit und Nicht-Reproduzierbarkeit und mithin ihre Funktionen für die stets umkämpfte Reproduktion gesellschaftlicher Macht“ zu studieren. ${ }^{2}$ Diesen „Kampf um die Reproduktionshoheit“ habe ich an anderer Stelle für das Musiksampling im Spannungsfeld von

1 Benjamin, Das Kunstwerk im Zeitalter seiner technischen Reproduzierbarkeit.

2 Schröter, Das Zeitalter der technischen Nicht-Reproduzierbarkeit, 29-30. 
Urheberrecht und Kreativität detailliert erforscht. ${ }^{3}$ Es liegt nahe, die Denkfigur nun auch auf den Bereich des akademischen Publikationswesens zu übertragen, um damit die dortige, urheberrechtlich geprägte Konfliktlinie adäquat beschreiben und analysieren zu können.

Denn mit jedem qualitativen Schub technischer Reproduzierbarkeit verändert sich das Verhältnis der beteiligten Akteur:innen: In der Regel entsteht ein Tipping Point, an dem das etablierte Akteursgefüge kippt und Neukonstellationen ermöglicht. So machte schon die gesteigerte technische Reproduzierbarkeit auf Grundlage des Buchdrucks im 15. Jahrhundert neue soziale und rechtliche Konfigurationen notwendig, die festlegten, wer welche Texte unter welchen Bedingungen kopieren durfte - eine Tätigkeit, die bis dahin handschriftlich erfolgte und vor allem von Mönchen in Klöstern erledigt wurde. Die Vokabel der sodann eingeführten, regional begrenzten „Druckprivilegien“ zeigt die Grundspannung der Reproduktionshoheit bereits an: Denn ein Privileg ist nur wenigen vorbehalten, die sich durch diese besondere Eigenschaft von den Nicht-Privilegierten unterscheiden, bestimmte Vorteile erlangen und ihre Privilegien meist zu verteidigen suchen. Das aus den Druckprivilegien hervorgegangene deutsche Urheberrecht steht etymologisch noch in dieser am Privileg orientierten Linie, denn es legt semantisch den Akzent auf die Bedürfnisse und Rechte der Urheber:in, also der Werkschöpfer:in. Im Gegensatz dazu deutet das angloamerikanische Copyright allein von seiner Wortwahl her mehr Offenheit an: Es betont das right to copy, also das Recht zu kopieren, welches sowohl Urheber:innen wie auch am eigentlichen Schöpfungsprozess Unbeteiligte adressiert.

Das Vervielfältigen von schriftlichen Erzeugnissen ist dabei nicht nur ein symbolisches Privileg, sondern auch eine materielle Ressource. Sie zeigt sich bereits in dem rechtlich-technischen Fakt, dass nur bestimmte Individuen und Organisationen Geldscheine herstellen und kopieren dürfen, wie Schröter bemerkt. So regeln technische Vorrichtungen in handelsüblichen Kopiergeräten die Reproduzierbarkeit von Geldscheinen und brechen automatisch den Kopiervorgang ab, wenn ein Geldschein auf der Scanauflage liegt. ${ }^{4}$ Die rechtlich abgesicherte Ressource der Reproduktionshoheit über Geldscheine wird also an eine Technologie delegiert, die unerlaubte Kopiervorgänge unterbricht.

Dieses Phänomen, Kopiervorgänge rechtlich und technisch einzuhegen, zu begrenzen und nur für bestimmte Gruppen zugänglich zu halten, be-

3 Fischer, Sampling in der Musikproduktion.

4 Schröter, Das Zeitalter der technischen Nicht-Reproduzierbarkeit, 14. 
zeichne ich als Suspension technischer Reproduzierbarkeit. ${ }^{5}$ Die Suspensionspraktiken verweisen also auf die Gegenmaßnahmen zu den Steigerungen technischer Reproduzierbarkeit. Die daraus entstehende Dynamik von Steigerung und Suspension ist hierbei von besonderem Interesse - genauso wie die daran anschließenden Praktiken der Unterlaufung und Umgehung der Suspensionsmaßnahmen. An diesem Set an Praktiken lässt sich die ständige Fortschreibung der Konfliktlinie gut erkennen und im Sinne wechselnder Kipppunkte interpretieren.

Für die Musikindustrie der 1950er und 1960er Jahre hat Monika Dommann einen solchen technisch-rechtlichen Kipppunkt sehr anschaulich herausgearbeitet. Sie zeigt anhand historischer Quellen, wie sich Verwertungsgesellschaften, Musiklabels und Technologiehersteller mit der Innovation des Tonbands mühsam auf neue urheberrechtliche Konfigurationen einigen mussten: Die signifikant gestiegene technische Reproduzierbarkeit, die es fortan auch Konsument:innen erlaubte, Musik und Klänge auf Tonband fixieren, kopieren und rearrangieren zu können, veränderte das Gefüge der beteiligten Parteien und musste daher kompensiert werden. ${ }^{6}$ Die daraus entstandene Leermedienabgabe ist bis heute eine stabile urheberrechtliche Konfiguration technischer Reproduzierbarkeit, die auch auf digitale Festplatten und andere Speichermedien Anwendung findet.

Mit dem Digitalen ergibt sich ein erneuter signifikanter Schub in der technischen Reproduzierbarkeit, der sich für das akademische Publikationswesen als Kipppunkt beschreiben lässt: So bringen Buchdruck und Tonband mit ihren mechanischen Prozessen noch verschiedene Einschränkungen mit sich, wie zum Beispiel den Verschleiß von Drucklettern oder Qualitätseinbußen bei Kopien von Kopien. Digitale Kopien hingegen ermöglichen unterschiedslose Exemplare des gleichen Werks, die beliebig oft vervielfältigt, ohne Qualitätsverlust und relativ kostengünstig verteilt werden können.

Für die über Jahrhunderte lang gewachsene und von urheberrechtlichen Maßgaben profitierende Verlagsindustrie stellt diese Steigerung der technischen Reproduzierbarkeit einen möglichen Kipppunkt dar, da als PDF vorliegende Publikationen von Konsument:innen selbst leicht verschickt, getauscht und zu abrufbaren Sammlungen akkumuliert werden können. Diese Entwicklung, die auch hinein in den Bereich des urheberrechtlich Illegalen führt, ist aus den Erfahrungen der Musikindustrie der frühen 2000er Jahre bekannt, in deren Folge nicht nur Abmahnpraktiken,

5 Fischer, Sampling in der Musikproduktion, 29.

6 Dommann Autoren und Apparate. 
technische Kopierschutzmechanismen und neue Angebote wie legale Streamingdienste eingeführt wurden, sondern auch beträchtliche Umsatzrückgänge zu verzeichnen waren. ${ }^{7}$ Für das akademische Publikationswesen indizieren Aufkommen und Stabilität von digitalen Schattenbibliotheken nun ebenfalls einen Kipppunkt in der medienindustriellen Entwicklung, welchen ich im Folgenden genauer darstelle.

\section{Aufstieg und Ausdifferenzierung akademischer Schattenbibliotheken}

In diesem Abschnitt stelle ich anhand von fünf verschiedenen Beispielen die Bandbreite akademischer Schattenbibliotheken dar und sorge für kontextualisierende Information. Die Beispiele haben miteinander gemeinsam, auf das gleiche Ziel hinzuarbeiten, nämlich eine hohe digitale Verfügbarkeit wissenschaftlicher Publikationen sicherzustellen. Sie unterscheiden sich aber hinsichtlich ihrer jeweiligen organisatorischen Verfasstheit und allgemeinen Sichtbarkeit. So ist das Hashtag \#canIhazPDF (Abschnitt 3.1) vor allem als praxeologisches Phänomen auf Twitter beobachtbar, während die vier anderen - Ubuweb, AAARG, Library Genesis und Science Hub (Abschnitte 3.2 und 3.3) - im engeren Sinne einer Schattenbibliothek fungieren. In allen Fällen jedoch agiert man mit urheberrechtlich mindestens fragwürdigen bis definitiv illegalen Praktiken, die von ideologisch-politischen Annahmen legitimiert und aus Effizienzgründen eingesetzt werden.

\section{1 \#IcanhazPDF als akademische Schattenpraxis}

Der Hashtag \#IcanhazPDF ist eine vergleichsweise niedrigschwellige Strategie, die für den Zugriff auf akademische Artikel Paywalls und andere Formen des Kopierschutzes umgeht. Der Hashtag wird auf Twitter verwendet: Die absichtlich falsche Schreibweise bezieht sich auf ein InternetMeme, das unter dem Slogan „Can I haz Cheeseburger” beziehungsweise "I can has Cheezburger” ab etwa 2008 bekannt wurde. In dem Meme geht es um eine Katze, die im Internet-Slang nach einem Cheeseburger verlangt. Es folgten viele weitere ähnliche Bilder, die auf diversen Internetplattformen geteilt und variiert wurden. Daraus resultierte eine virale Dy-

7 Dolata Berliner Journal für Soziologie 2008, 344; Sinnreich, Mashed up. 
namik, in der sich der Cheeseburger-Slogan zunehmend von seinem Original entfernte und für andere Kontexte nutzbar wurde. ${ }^{8}$

So übertrug die US-amerikanische Kognitionswissenschaftlerin Andrea Kuszewski den Slogan 2011 auf die Suche nach akademischen Artikeln. Sie kreierte den Hashtag \#IcanhazPDF und setzte damit auf Twitter eine digitale Bewegung in Gang: Nutzer:innen können unter Verwendung des Hashtags und Angabe des gesuchten Artikels sowie der eigenen Emailadresse Artikel anfragen, die andere Nutzer:innen für sie herunterladen und Ihnen zusenden, sofern ihre heimischen Universitätsbibliotheken einen Zugang dazu erworben haben.

Die Praxis um den Hashtag appelliert an den Gemeinschaftsgedanken der global vernetzten Wissenschaft und erweitert die Praxis unter Forscher:innen, sich per Email gegenseitig Literatur zu empfehlen und zuzusenden. Gleichzeitig findet \#IcanhazPDF zu Teilen im Schatten statt: Da das Versenden der Artikel in den allermeisten Fällen Urheberrechte verletzt, gehört es zur Praxis, sich nicht öffentlich bei den Sender:innen zu bedanken. Dieser Quellenschutz ist Kuszewski zufolge ein wichtiger Teil von \#IcanhazPDF und zeigt in Form einer ethischen Verpflichtung die allgemeine Sorge vor dem Abmahnwesen der akademischen Großverlage. ${ }^{9}$ Dem entspricht auch die Aufforderung, den Anfrage-Tweet nach dem erfolgreichen Erhalt des Artikels wieder zu löschen. ${ }^{10}$

Diese Aufforderung ist im Profil des Twitterbots @hecanhazpdf als Verhaltensregel notiert („etiquette reminder“), ${ }^{11}$ der sämtliche Tweets unter dem Hashtag automatisch seinen aktuell etwas mehr als 1000 Follower:innen ausspielt (Stand: Juli 2020). ${ }^{12}$ Vermutlich abonnieren noch zahlreiche weitere Twitternutzer:innen den Hashtag, um nach den Prinzipien von Tauschbörsen ${ }^{13}$ und Gabentausch im Kreise Gleichgesinnter zu partizipie-

8 Fischer/Grünewald-Schukalla, kommunikation @ gesellschaft 2018, 1, 3.

9 Mohdin/Quartz, How to Get Free Access to Academic Papers on Twitter, https://w ww.theatlantic.com/technology/archive/2015/10/why-some-academics-are-sharingtheir-papers-for-free/411934/ (zuletzt abgerufen am 03.08.2020).

10 Gardner/Gardner, Bypassing Interlibrary Loan Via Twitter: An Exploration of \#icanhazpdf Requests (Conference Paper), http://www.ala.org/acrl/sites/ala.org.ac $\mathrm{rl} /$ files/content/conferences/confsand preconfs/2015/Gardner.pdf (zuletzt abgerufen am 03.08.2020), 96.

11 shecanhazPDF, \#icanhazpdf etiquette reminder: https://twitter.com/hecanhazpdf/s tatus/598391209749516288 (zuletzt abgerufen am 03.08.2020).

12 shecanhazPDF, Twitter Profile, https://twitter.com/hecanhazpdf (zuletzt abgerufen am 03.08.2020).

13 Krömer/Sen, No Copy. 
ren. ${ }^{14}$ \#IcanhazPDF ist nicht auf bestimmte Fachgrenzen beschränkt, sondern erfasst prinzipiell alle akademischen Disziplinen, geografischen Herkunftsorte und Jahrgänge der gesuchten Literatur. Insofern ist der Hashtag auch ein interessantes Werkzeug, um stichprobenartig einen Einblick in die Forschung und Lesegewohnheiten der jeweiligen Wissenschaftler:innen zu erhalten: Denn durch die Nutzung des Hashtags zeigen Forscher:innen, welche Literatur sie gerade rezipieren oder zumindest rezipieren wollen. Innerhalb der Social Media-Strukturen sorgen Forscher:innen durch die willentliche oder nicht-willentliche Ausstellung ihres Konsums also für eine partielle Öffnung des Produktionsprozesses wissenschaftlicher Forschung, der sonst vor allem durch Abgeschlossenheit geprägt ist.

Die Anfrage- und Bereitstellungspraktiken um \#IcanhazPDF sind Ausdruck allgemeinerer Phänomene der internetbasierten Vernetzung und der digitalen Reorganisation des wissenschaftlichen Gütertauschs. ${ }^{15}$ Denn \#IcanhazPDF bedeutet ja nicht nur die Unterlaufung urheberrechtlicher Kontroll- und Verwertungsmechanismen der akademischen Großverlage, sondern auch die Umgehung von Bibliotheksdienstleistungen wie Fernleihe und anderen Recherche- und Beschaffungsmaßnahmen, die bis vor wenigen Jahren exklusiv von Bibliothekar:innen durchgeführt wurden. Die Vokabel „Fernleihe” impliziert dabei noch die physische Einzigartigkeit von Büchern, die erst aus einer fremden Bibliothek ausgeliehen, kopiert und zurückgegeben werden. Diese Qualität von rivalen Gütern und entsprechende Nutzungspraktiken würden mit digitalen Kopien eigentlich obsolet werden und ein Ende der Knappheit bedeuten; teilweise gibt es jedoch noch Nutzungsangebote, in denen eine digitale Buchkopie nur zeitlich begrenzt zur Verfügung steht. Viele Bibliotheken verlangen für ihren Fernleihe-Service ein Entgelt, das insbesondere Studierende oder NichtAngehörige der jeweiligen Universitäten nicht in allen Fällen zahlen können oder möchten. Man muss dazu auch betonen, dass der Inhalt der gewünschten Artikel zum Zeitpunkt der Bestellung in der Regel noch nicht bekannt ist und für eine Forschungsarbeit mitunter dutzende oder sogar hunderte Artikel gesichtet werden.

Durch die Praxis um \#IcanhazPDF verlagert sich die Literaturbeschaffung weg von den offiziellen, eigentlich dafür vorgesehenen Distributionskanälen der Bibliotheken in Richtung einzelner Wissenschaftler:innen. Diese können ihren oftmals unbekannten Kolleg:innen aus möglicherweise altruistischen Motiven einen Gefallen tun, Gemeinschaftspflege betrei-

14 Schulz-Schaeffer KZfSS 2017, 27, 31.

15 Karaganis, Introduction, in: Karaganis: Shadow Libraries, 1, 11. 
ben und ihrerseits auf reziproke Tauschgeschäfte hoffen. Gleichzeitig verringern sie die monetären Kosten der Beschaffung und stellen die Artikel von Anfang an digital zur Verfügung, was bei der klassischen Fernleihe der Bibliotheken nicht gesichert ist. \#IcanhazPDF kann daher in einigen Fällen als Akt zivilen Ungehorsams verstanden werden, mit dem die für die Autor:innen und Bibliotheken dysfunktionalen urheberrechtlichen Strukturen der Großverlage problematisiert und unterlaufen werden. In vielen anderen Fällen mag die Nutzung einfach nur praktisch und nicht ideologisch motiviert sein, da das Versenden von PDFs schnell geschieht. Die Praxis ist damit auch keine Schattenbibliothek im engeren Sinne, sondern eher ein aus Effizienzgründen ad hoc entwickeltes Mittel der Beschaffung, das von den Nutzer:innen aus Angst vor Abmahnungen selbst in den Schatten getragen wird. Dass der Schatten nicht selbstverständlich ist, sondern bei jeder Anfrage erneut reproduziert werden muss, zeigen die Aufforderungen, Anfragetweets zu löschen und sich bei den Sender:innen nicht zu bedanken.

\subsection{Thematisch spezialisierte Schattenbibliotheken: UbuWeb und AAARG}

Neben der Praxis um \#IcanhazPDF haben sich in den letzten Jahren eine Reihe von Internetplattformen etabliert, die als Schattenbibliotheken im engeren Sinn charakterisiert werden können und einen höheren Organisationsgrad aufweisen: UbuWeb und AAARG sowie LibGen und SciHub fungieren als urheberrechtlich fragwürdige bis illegale Plattformen. Sie stellen genauso wie klassische, auf Langfristigkeit angelegte Bibliotheken wissenschaftliche und literarische Werke, Artikel und Bücher komplett oder in Auszügen als PDFs zur Verfügung. Die vier genannten unterscheiden sich hinsichtlich ihres Organisationsgrads und ihrer politisch-intellektuellen Ausrichtung, sowie hinsichtlich der Frage, wie sehr sie im Schatten der öffentlichen Aufmerksamkeit stehen und in versteckten Bereichen agieren. UbuWeb und AAARG sind thematisch spezialisiert, weswegen ich sie in diesem Abschnitt bespreche. LibGen und SciHub dagegen sind umfassend orientiert und decken alle Fachgebiete ab (Abschnitt 3.3).

UbuWeb wurde in der zweiten Hälfte der 1990er Jahre von dem USamerikanischen Künstler Kenneth Goldsmith zunächst als Plattform für Poesie und avantgardistische Literaturformen gegründet, wenig später auch für digitale Musik- und Videodateien geöffnet. Goldsmith erkannte die Notwendigkeit und gesellschaftliche Nachfrage eines digitalen Archivs für avantgardistische Kunstformen und weitere Dokumente, die sonst nur analog vorlägen und damit in der damals gerade erst anbrechenden digita- 
len Ära des Internets nicht auffindbar, also quasi non-existent wären. Auf UbuWeb, nach eigener Darstellung der „Robin Hood of the Avant-garde“, sammelt, dokumentiert, kuratiert, veröffentlicht und archiviert Goldsmith diverse Ausdrucksformen in Wort, Ton und Bild, darunter auch Reden von Politiker:innen, Gedichte, akademische Essays, alte Radiosendungen und vieles weitere. Der Website zufolge veröffentlicht Goldsmith jeden Tag eine neue Datei auf UbuWeb; es gibt dort auch Abteilungen, die von anderen Künstler:innen bespielt werden. Zudem arbeiten Ehrenamtliche für UbuWeb, die beispielsweise Texte einscannen und online stellen. ${ }^{16}$

Goldsmith betont in einem eigenen Buch, das er in Traditionsanschluss an avantgardistische Strömungen mit „Duchamp is my Lawyer" betitelt, seinen Anspruch auf allgemein gesicherte Zugänglichkeit der Dokumente. Die Seite ist absichtlich low tech gestaltet, nach wie vor in HTML programmiert und möglichst simpel gehalten, so dass sie theoretisch auch offline geöffnet werden kann - zum Beispiel von einem USB-Stick, auf dem die Daten der Website gespeichert sind. Eine Abhängigkeit von Servern Dritter lehnt UbuWeb ab, dementsprechend verzichtet die Seite im Wesentlichen auf eingebettete Streams oder ähnliches. Man will, wohl auch im Falle urheberrechtlicher Abmahnungen, Unabhängigkeit und Arbeitsfähigkeit bewahren. Die Wiederherstellbarkeit der Website ist durch Mirrors aus dem Umfeld von UbuWeb gesichert „so that even if UbuWeb should disappear, it will never disappear." ${ }^{17}$ Neben der postmodernen Urheberrechtskritik verfolgt UbuWeb das Projekt, finanziell prekäre Bibliotheksstrukturen aufzuzeigen und durch die offensiv hergestellten Zugangsmöglichkeiten zu problematisieren. Gleichzeitig lässt sich UbuWeb selbst als postmodernes Kunstprojekt verstehen, in dem das Nebeneinander der verschiedenen Dokumente neue Querbezüge ermöglicht und tradierte Ordnungen und Narrative in Frage stellt.

Während UbuWeb frei zugänglich ist und aufgrund seines kritischen Ansatzes eher das Problematische am Verhältnis von Archivierung und Urheberrechten in den Fokus rückt und es dadurch gewissermaßen ästhetisiert, versteht sich die Plattform AAARG eher als edukatives, autodidaktisches Instrument. Für die Plattformen werden allerdings Einladung und Registrierung benötigt. Die Abkürzung AAARG steht für „Artists, Architects und Activists Reading Group“, was den intellektuellen Anspruch und gleichzeitig den organisatorischen Ansatz des Projekts bereits umschreibt:

16 UbuWeb, About UbuWeb, http://www.ubu.com/resources/index.html (zuletzt abgerufen am 03.08.2020).

17 Goldsmith, Duchamp is my Lawyer, 22. 
Eine Gruppierung, die eine Grenze zwischen drinnen und draußen, zwischen Mitgliedern und Nicht-Mitgliedern zieht, wohl auch um urheberrechtlichen Abmahnungen vorzubeugen. ${ }^{18}$

2005 als digitale Bibliothek und Plattform für Studierende und andere intellektuell Interessierte in Los Angeles gegründet, die Texte miteinander austauschen, diskutieren und sammeln wollten, wurde AAARG zunehmend international und von weiteren Gruppen genutzt. Das Prinzip Lesekreis wurde mit AAARG ins Digitale übersetzt und weiterentwickelt. AAARG bietet seinen registrierten Mitgliedern vor allem Texte der Kritischen Theorie, postmoderne und poststrukturalistische Philosophien und kulturwissenschaftliche Abhandlungen aus den Bereichen Kunst, Film, Architektur und Literatur. Die Benutzer:innen stellen eigene Dateien, darunter auch selbst angefertigte Scans von Aufsätzen oder ganzen Büchern bereit. Auf der Website lassen sich Texte suchen, herunterladen und anfragen, sowie in thematische Listen (sogenannte „issues“) organisieren und kommentieren. Die verschiedenen, kollaborativ ausgerichteten Funktionen sollen, so die Idee des Projekts, das selbständige, autodidaktische Lernen außerhalb der klassischen Bildungsinstitutionen ermöglichen und fördern. Aufgrund seiner laxen urheberrechtlichen Politik erhielt die Plattform bereits einige Klagen und rechtliche Probleme, verhielt sich aber stets konziliant und löschte die angemahnten Dateien. Um die Auffindbarkeit für Behörden zu erschweren und Kontrollen zu umgehen, änderte die Seite mehrfach ihre eigene URL, beispielsweise durch Hinzufügung weiterer "A"s und dem Wechsel der Top-Level-Domain. Aktuell ist sie unter aaaaarg.fail zu erreichen (Stand: Mai 2020).

\subsection{Umfassende Schattenbibliotheken: LibGen und SciHub}

Während UbuWeb und AAARG aufgrund thematischer und ideologischer Fokusse noch relativ kleine Korpora anbieten, haben sich mit den russischen Plattformen LibGen und SciHub zwei Großanbieterinnen positioniert, die auf umfassende Abdeckung zielen. LibGen - kurz für Library Genesis - lässt sich als aggregierte Schattenbibliothek charakterisieren, da sie aus der Zusammenführung einiger größerer und kleinerer Textsammlungen in Russland ab 2008 hervorging. Als originär russische Schattenbibliothek enthielt der Katalog von LibGen zunächst vor allem russischsprachige

18 Ekström, The Illicit Information Community, http://uu.diva-portal.org/smash/get/ diva2:1143483/FULLTEXT01.pdf (zuletzt abgerufen am 03.08.2020). 
Texte; später kamen auch Texte in Sprachen wie Englisch, Spanisch, Deutsch, Französisch, Chinesisch und weitere hinzu. Zuwächse erfolgen meist sprunghaft durch die Hinzufügung von massenhaften Textsammlungen, die durch automatisierte Downloads von Großverlagen, Repositorien oder anderen massenweisen Leaks über Universitätsnetzwerke („Biblioleaks“) zustande kommen. ${ }^{19}$ Neben wissenschaftlicher Literatur sind auch Comics, Romane, Magazine und weitere Textsorten bei LibGen abrufbar. Im Januar 2014 hostete die Plattform etwa 25 Millionen Dokumente. ${ }^{20}$

Dass LibGen in Russland entstand und sich etablierte, ist kein Zufall: Einerseits bietet die laxe Haltung des russischen Staats gegenüber urheberrechtlichen Verletzungen im Mediensektor ${ }^{21}$ eine ideale Bedingung für Schattenbibliotheken; auf der anderen Seite besitzt die russische Bevölkerung aufgrund staatlicher Mangelwirtschaft und Zensur aus der Sowjetzeit umfassende Erfahrung im Agieren im Untergrund und im Tausch knapper Güter. Dies zeigte sich im postsowjetischen Russland der 1990er und 2000er, als wissenschaftliche Literatur aus dem Westen eine zunehmend gefragte Ressource für die russische Forschung wurde, aber trotzdem schwer zu beschaffen war. Digitalisierung und internetbasierte Vernetzung schafften Abhilfe für dieses Problem, da Texte als digitale Dateien nun beliebig oft vervielfältigbar und ohne Qualitätsverlust an unterschiedlichen Orten speicherbar wurden, was ein prinzipielles Ende der Knappheit markierte. ${ }^{22}$

Vor diesem Hintergrund erklärt sich auch der Anspruch von LibGen nach umfassender Abdeckung, der sich neben der schieren Korpusgröße auch in der programmiertechnischen Offenheit zeigt: Dank des offenen Codes kann und soll die Schattenbibliothek als Ganze von anderen Nutzer:innen heruntergeladen, kopiert und unter neuer URL wieder der Öffentlichkeit zur Verfügung gestellt werden (ähnlich wie UbuWeb). Mithilfe technischer Reproduzierbarkeit hält sich LibGen über verschiedene unabhängige Mirrors selbst am Leben und stellt seinen urheberrechtlich zwar problematischen, aber trotzdem stark nachgefragten, Service langfristig sicher.

Mit LibGen verbunden ist die Schattenbibliothek SciHub (Langform: Science Hub), die 2011 von der Kasachin Alexandra Elbakyan gegründet wurde. Laut eigener Beschreibung war Elbakyan als Promotionsstudentin

19 Bodó The Genesis of Library Genesis, in: Karaganis, Shadow Libraries, 25, 28.

20 Cabanac JASIST 2016, 874, 874.

21 Lobato Shadow Economies of Cinema, 48 und 69.

22 Bodó The Genesis of Library Genesis, in: Karaganis, Shadow Libraries, 25, 31. 
mit dem Problem konfrontiert, von ihrem Heimatort in Kasachstan nicht auf die wissenschaftlichen Artikel zugreifen zu können, die sie für ihre Dissertation benötigte. Die Paywalls der wissenschaftlichen Großverlage ließen eine Auseinandersetzung mit der gewünschten Forschung aus monetären Gründen nicht zu und ihre Universitätsbibliothek konnte aufgrund fehlender Abonnements keinen Zugang zu den Artikeln herstellen. Aus dieser Frustration heraus entwickelte Elbakyan SciHub, um einen einfachen, allgemein verfügbaren Zugang zu sämtlichen, nicht per Open Access verfügbaren wissenschaftlichen Artikeln der Großverlage herzustellen. ${ }^{23}$ SciHub kooperiert mit LibGen, das als Mirror fungiert, um die Publikationen langfristig zu archivieren und den Zugang zu ihnen zu gewährleisten. Über SciHub sind derzeit mehr als 74 Millionen Dokumente abrufbar. ${ }^{24}$

Die Funktionsweise von SciHub lässt sich als automatisierte, technisch delegierte Umgehungsstrategie beschreiben: Für den Zugriff auf einen Artikel wird dem Verlag die IP einer Bibliothek vorgegaukelt, die den $\mathrm{Zu}$ gang zu dem betreffenden Werk bereits erworben hat - auch wenn die Nutzer:in eigentlich von anderer Stelle zugreift. Auf diese Weise werden die in harte Technik delegierten urheberrechtlichen Konfigurationen unterlaufen. SciHub operiert auch in anderer Weise mit Umgehungsstrategien, es kommt zu einem regelrechten „Katz-und-Maus“-Spiel, das bereits aus dem medienindustriellen Komplex der Unterhaltungsindustrie und seinem Kampf gegen Online-Piraterie bekannt ist: So wechselt SciHub in unregelmäßiger Regelmäßigkeit seine eigene IP sowie seine Toplevel-Domain, um Kontrollen und Blockierungen durch Service Provider zu erschweren. ${ }^{25}$ In Deutschland ist die Website sci-hub.tw beim deutschen Internetanbieter Vodafone gesperrt; ruft man sie auf, erscheint der Hinweis: „Dieses Portal ist aufgrund eines urheberrechtlichen Anspruchs vorläufig nicht verfügbar. “26 Über sci-hub.se ist die Seite jedoch zum gleichen Zeitpunkt problemlos erreichbar. ${ }^{27} \mathrm{Im}$ staatlich kontrollierten Internet von China scheint SciHub beziehungsweise ein SciHub-Klon ebenfalls verfügbar zu sein. ${ }^{28}$ Bemerkenswert ist zudem das Angebot @scihubbot auf der

23 Bohannon Science 2016, 508, 511.; Seer LIBREAS. Library Ideas 2017, 1, 2.

24 Strecker LIBREAS. Library Ideas 2019, 1, 1.

25 lovescibub, Love Science, Love Sci-Hub!, https://lovescihub.wordpress.com (zuletzt abgerufen am 03.08.2020).

26 Vodafone, https://www.vodafone.de/webblock.html (zuletzt abgerufen am 03.08.2020).

27 Sci-Hub, https://sci-hub.se (zuletzt abgerufen am 03.08.2020).

28 企我论文键下载, http://doi.qqsci.com/ (zuletzt abgerufen am 03.08.2020). 
Messenger-Plattform Telegram. ${ }^{29}$ Telegram ist neben seiner harmlosen Kurznachrichtenfunktion zu einem etablierten digitalen Umschlagsplatz für Drogen, Waffen, Verschwörungstheorien, rechtsextreme oder ISIS-Propaganda geworden. Das „Dark Social“, wie der Messengerdienst aufgrund seiner laxen Kontrolle auch genannt wird, macht damit dem Darknet Konkurrenz. ${ }^{30}$ Das zeigt, dass SciHub ähnliche Wege einschlägt wie andere illegale, im Schatten liegende Geschäftszweige, um arbeitsfähig zu bleiben.

In der urheberrechtlichen Illegalität operiert SciHub also mit einigen Kniffen, um den Zugang zu akademischen Publikationen für die Allgemeinheit aufrechtzuerhalten. Im Vergleich zu den anderen Schattenbibliotheken ist SciHub wohl die raffinierteste Lösung: sie erzwingt für Forscher:innen Spielräume, ohne aber das dahinter liegende Problem auflösen zu können. Das radikale wie gewitzte Mittel, massenhaften Zugang zu Publikationen über IP-Verschleierung und Umgehung der Kontrolle herzustellen, entspricht der geschäftigen Kreativität der Verlage, ganz bewusst Lock-In-Effekte durch technologisch gehärtete Urheberrechtsmechanismen zu erzielen, um die eigene Reproduktionshoheit zu sichern und Preisdiktate durchzusetzen.

\section{Schattenbibliotheken als Indikator für kippende Akteursgefüge}

Der kursorische Überblick zeigt, dass das Geschäftsmodell der bisher dominierenden akademischen Großverlage ernstzunehmende Konkurrenz erhalten hat und ins Wanken geraten könnte. Ein Tipping Point ist demnach nicht nur in Sicht, sondern aktuell seit einigen Jahren voll im Gange. Denn die Demokratisierung der Publikationsmittel aufgrund gesteigerter technischer Reproduzierbarkeit hat dazu geführt, dass sich eine Reihe an Schattenbibliotheken und -praktiken mit verschiedenen Organisationsgraden entwickelt und etabliert hat, mit denen das digitale Kopieren wissenschaftlicher Artikel einfach, billig und effizient verläuft - nicht nur in abseitigen, schwer zugänglichen Kanälen, sondern für Wissenschafler:innen leicht erreich- und bedienbar.

Zwar greifen viele Forscher:innen nach wie vor hauptsächlich auf die bewährten Angebote und Literaturbeschaffungsmaßnahmen ihrer Bibliotheken zurück, die auch zukünftig ihre Berechtigung haben werden -

29 Telegram, https://telegram.me/scihubbot (zuletzt abgerufen am 03.08.2020).

30 Vice, Themenschwerpunkt Telegram: https://www.vice.com/de/topic/telegram (zuletzt abgerufen am 03.08.2020). 
doch sind mit digitaler Vernetzung und Internationalisierung der Wissenschaft auch Forscher:innen und Bibliotheken involviert, für die der legale kostenpflichtige Zugang zu wissenschaftlicher Literatur unerschwinglich bleibt. Gleiches gilt für Studierende, die Kopierschutzmaßnahmen von wissenschaftlicher Literatur als behindernd und nicht nachvollziehbar empfinden, da der Zugang zu digitalen Medieninhalten für sie selbstverständlicher Alltag ist. Und: Die gegenwärtigen Studierenden sind die Forscher:innen und Professor:innen der Zukunft, was das Akzeptanzproblem sicherlich weiter verschärfen wird.

Der ideologische Überbau der jeweiligen Schattenbibliotheken scheint für die Konsument:innen nachrangig zu sein: Was für sie zählt, ist, dass wissenschaftliche Literatur schnell, unkompliziert und kostengünstig erreicht und gegebenenfalls weiter distribuiert werden kann. An diesem Bedarf orientieren sich Schattenbibliotheken, während die regulären Anbieterinnen, also die Großverlage, mit Zugangsbarrieren, Kopierschutzmechanismen und Wucherpreisen aufwarten, um ihre urheberrechtlich gesicherte Reproduktionshoheit auszuschöpfen. Die Großverlage agieren jedoch am Bedarf vorbei: Sie können oder wollen die vertriebene Literatur nicht in der nachgefragten Form und zum passenden Preis zur Verfügung stellen.

Dass Schattenbibliotheken ihre Präsenz mitunter selbst in schattige und teils illegale Bereiche verlagern, zeigt, dass der gesellschaftliche Bedarf nach frei zugänglicher wissenschaftlicher Literatur enorm groß ist - so groß, dass er nicht ohne Weiteres unterdrückt oder vollständig kontrolliert werden kann, sondern in illegale Bereiche abwandert und sich in Umgehungsstrategien kanalisiert. Narrativ gestützt werden Schattenbibliotheken von ihren Gründer:innen und Betreiber:innen mit Gründungserzählungen, die das Widerständige der untergründigen Guerilla-Praxis betonen. Der Kampf zwischen den kleinen, wendigen und technisch überlegenen Schattenbibliotheken, die Urheberrechtspiraterie für den guten Zweck betreiben, und den Großverlagen, die dabei meist als träge, profitsüchtig und aversiv gegenüber Innovationen dargestellt werden, entspricht dieser Erzählung. Doch das beschriebene Narrativ hat noch eine weitere Facette, die über die üblichen Urheberrechtspiraterie-Erzählungen hinausgeht: Im Gegensatz zu den Filesharing-Plattformen für Musik und Film der frühen 2000er Jahre geht es bei akademischen Schattenbibliotheken um die Rückeroberung von Produkten, die als Eigentum empfunden werden: Einerseits weil die Produktion der wissenschaftlichen Texte in der Regel mit öffentlichen Mitteln finanziert wurde und wissenschaftliches Wissen daher als allgemeines, öffentliches Gut verstanden wird; andererseits weil Wissenschaftler:innen, darunter teilweise die Autor:innen selbst, oft keinen 
kostenlosen Zugriff auf die gewünschten Artikel erhalten, sondern diese im schlechtesten Falle zurückkaufen müssen und damit von ihren eigenen Produkten entfremdet werden.

Daneben indizieren Genese, Stabilität und Ausdifferenzierung der verschiedenen, mit Umgehungskreativität operierenden Schattenbibliotheken, wie prekär die Knappheit von digitalen Dateien im wissenschaftlichen Publikationswesen real eigentlich ist. Denn wenn Verlage diese Knappheit nicht künstlich herstellen und kontrollieren würden, gäbe es sie gar nicht. Hier tritt eine gewaltige Konfliktlinie zutage, an der sich ablesen lässt, dass sich die bisherigen Zugangsbeschränkungen nicht mehr mit der enorm gestiegenen technischen Reproduzierbarkeit in Deckung bringen lassen. Diese Konfliktlinie hat sich nicht kurzfristig aufgebaut, sondern über Jahrzehnte hinweg gebildet, wenngleich das bisherige Akteursgefüge und die damit verbundenen urheberrechtlichen Konfigurationen offensichtlich funktional waren oder zumindest aus Mangel an Alternativen weithin akzeptiert wurden. Dass einzelne Menschen nun ganze Bibliotheken mit mehreren Millionen Dokumenten aufbauen und anderen Menschen zur Verfügung stellen können, war innerhalb des bisherigen Gefüges nicht vorgesehen. Entsprechend heftig fällt nun der Kampf um die Reproduktionshoheit in der akademischen Medienindustrie aus und es ist nicht zu erwarten, dass die Geschütze in beiden Lagern demnächst abgebaut werden.

\section{Literatur}

Benjamin, Walter, Das Kunstwerk im Zeitalter seiner technischen Reproduzierbarkeit. Drei Studien zur Kunstsoziologie, Frankfurt a.M. 2012 [1935].

Bodó, Balazs, Libraries in the Post-Scarcity era, in: Porsdam, Helle (Hrsg.), Copyrighting Creativity: Creative Values, Cultural Heritage Institutions and Systems of Intellectual Property, Ashgate 2015, S. 75-92.

Bodó, Balasz, The Genesis of Library Genesis, in: Karaganis, Joe (Hrsg.), Shadow Libraries: Access to Knowledge in Global Higher Education, The MIT Press 2018, S. 25-52.

Bohannon, John: Who's downloading pirated papers? Everyone. Science 352 (2016) S. 508-512.

Cabanac, Guillaume: Bibliogifts in LibGen? A study of a text-sharing platform driven by biblioleaks and crowdsourcing. Journal of the Association for Information Science and Technology 67 (2016) S. 874.

Dolata, Ulrich: Das Internet und die Transformation der Musikindustrie. Berliner Journal für Soziologie 18 (2008) S. 344. 
Ekström, Björn, The Illicit Information Community: Information - Practical Reflections on the Shadow Library AAARG, in: Arvola, Paavo et al. (Hrsg.) Improving Quality of Life Through Information: Proceedings of the XXV Bobcatsss Symposium, 2017 Tampere, S. 121-125.

Fischer, Georg, Sampling in der Musikproduktion. Das Spannungsfeld zwischen Urheberrecht und Kreativität, Marburg 2020.

Fischer, Georg / Grünewald-Schukalla, Lorenz (Hrsg.): Originalität und Viralität von (Internet-)Memes, kommunikation @ gesellschaft (Sonderausgabe), 2018.

Gardner, Carolyn / Gardner Gabriel Bypassing Interlibrary Loan Via Twitter: An Exploration of \#icanhazpdf Requests, http://www.ala.org/acrl/sites/ala.org.acrl/files /content/conferences/confsandpreconfs/2015/Gardner.pdf (zuletzt abgerufen am 03.08.2020).

Goldsmith, Kenneth, Duchamp is my Lawyer. The Polemics, Pragmatics and Poetics of UbuWeb, New York City 2020.

Karaganis, Joe, Introduction: Access from Above, Access from Below, in: Karaganis, Joe (Hrsg.). Shadow Libraries: Access to Knowledge in Global Higher Education, The MIT Press 2018, S. 1-24.

Krömer, Jan / Sen, Evrim, No Copy. Die Welt der digitalen Raubkopie, Leipzig 2006.

Lobato, Ramon, Shadow Economies of Cinema: Mapping Informal Film Distribution, London 2012.

lovescihub, Love Science, Love Sci-Hub!, https://lovescihub.wordpress.com (zuletzt abgerufen am 03.08.2020).

Mohdin, Aamna / Quartz: How to Get Free Access to Academic Papers on Twitter (The Atlantic), https:/www.theatlantic.com/technology/archive/2015/10/why-so me-academics-are-sharing-their-papers-for-free/411934/ (zuletzt abgerufen am 03.08.2020).

Schröter, Jens: Das Zeitalter der technischen Nicht-Reproduzierbarkeit, Navigationen 10 (2010), S. 9.

Schulz-Schaeffer, Ingo: Crowdsupporting als Gabentausch. Zur soziologischen Analyse des Crowdfunding, Kölner Zeitschrift für Soziologie 69 (2017), S. 27.

Sci-Hub, https://sci-hub.se (zuletzt abgerufen am 03.08.2020).

shecanhazPDF, \#icanhazpdf etiquette reminder: https://twitter.com/hecanhazpdf/sta tus/598391209749516288 (zuletzt abgerufen am 03.08.2020).

shecanhazPDF, Twitter Profile, https://twitter.com/hecanhazpdf (zuletzt abgerufen am 03.08.2020).

Seer, Veil: Von der Schattenbibliothek zum Forschungskorpus. Ein Gespräch über Sci-Hub und die Folgen für die Wissenschaft, LIBREAS. Library Ideas 32 (2017), S. 1.

Sinnreich, Adam, Mashed up. Music, Technology, and the Rise of Configurable Technology, Amherst/Massachusetts 2010.

Steinhauer, Eric: Die Nutzung einer "Schattenbibliothek“ im Licht des Urheberrechts, LIBREAS. Library Ideas 30 (2016), S. 128. 
Strecker, Dorothea: Nutzung der Schattenbibliothek Sci-Hub in Deutschland, LIBREAS Library Ideas (36) 2019, S. 1.

Telegram, https://telegram.me/scihubbot (zuletzt abgerufen am 03.08.2020).

UbuWeb, About UbuWeb, http://www.ubu.com/resources/index.html (zuletzt abgerufen am 03.08.2020).

Vice, Themenschwerpunkt Telegram: https://www.vice.com/de/topic/telegram (zuletzt abgerufen am 03.08.2020).

Vodafone, https://www.vodafone.de/webblock.html (zuletzt abgerufen am 03.08.2020).

企我论文键下载, http://doi.qqsci.com/ (zuletzt abgerufen am 03.08.2020). 